\title{
Improving vaccines by targeting antigens to dendritic cells
}

\author{
Ken Shortman ${ }^{1}$, Mireille H. Lahoud \\ and Irina Caminschi
}

The Walter and Eliza Hall Institute of Medical Research

Parkville Victoria 3050, Australia

${ }^{1}$ Corresponding author: Tel, 61-3-9345-2531;

E-mail, shortman @wehi.edu.au

DOI 10.3858/emm.2009.41.2.008

Accepted 20 January 2009

Abbreviations: $\mathrm{CDC}$, conventional $\mathrm{DC}$; $\mathrm{DC}$, dendritic cell/cells; $\mathrm{pDC}$, plasmacytoid DC

\begin{abstract}
A new approach to enhancing the effectiveness of vaccines is to deliver antigens selectively to dendritic cells (DC) in situ, via monoclonal antibodies specific for particular DC surface molecules. This can markedly enhance CTL responses and, via helper T cells, also enhance antibody responses. DC activation agents or adjuvants must also be administered for effective CTL responses, but in some cases good antibody responses can be obtained without adjuvants. Here we review the role of different DC subsets and different DC target molecules in obtaining enhanced immune responses.
\end{abstract}

Keywords: adjuvants, immunologic; antibody formation; CLEC9a protein, human; DEC-205 receptor; dendritic cells; vaccines

\section{Introduction}

Dendritic cells (DC) are the crucial sentinel cells of the adaptive immune system (Steinman, 1991). They continuously sample the antigenic environment and are sensors of microbial invasion or tissue damage. They not only process and present antigens to activate naïve $T$ lymphocytes, but they also regulate the nature of the $T$ cell response obtained, determining whether it leads to tolerance or to a Th1 or Th2 effector T cell responses. These central roles make manipulation of the DC system an attractive strategy for modulating immune responses.

It is now clear that one of the roles of adjuvants which enhance response to antigens is to activate the DC system; DC themselves have been termed
"Nature's Adjuvants". DC have been used in a very direct way in the clinic for the immunotherapy of cancer, so far with only limited success. In this approach DC are produced in culture from the patient's progenitor cells, the cultured DC are loaded with tumor antigens and then injected back into the patient, in the hope of inducing a more effective anti-tumour response (Tacken et al., 2007). Even if further refinements increase the success of this approach, it remains complex and expensive. The alternative considered in this review is to target antigens directly to the DC in situ, with or without associated DC activation agents. The current approach uses monoclonal antibodies (mAb) specific for molecules on the DC surface to carry linked antigens directly to the DC (Tacken et al., 2007). In principle this should reduce the quantity of antigen needed and improve the effectiveness of the vaccine injected. We are particularly interested in enhancing antibody responses by this approach, so that is the main focus of this review.

\section{DC subtypes}

Before considering targeting antigens to DC in situ, it is important to know about the types of DC which will receive the antigen cargo. The DC normally used for current immune therapy are derived from monocytes stimulated with GM-CSF and IL-4, which models "inflammatory DC", not normally found in steady-state but produced in vivo as a response to inflammation (Shortman and Naik, 2007). As an emergency response DC, they may well be appropriate for such DC transfer therapy, but they are not the type of DC which would immediately encounter a targeted vaccine antigen injected into a healthy individual. Several distinct types of DC, differing in origin and specialised functions, are present in steady-state (Shortman and Naik, 2007). One major division is into plasmacytoid DC ( $\mathrm{pDC}$, sometimes called lymphoid $D C$ ) and conventional DC (CDC, sometimes called myeloid DC). The pDC serve as the major producers of type 1 interferons in response to viral infections, and only assume a dendritic form and antigen-presenting functions after activation; CDC already have dendritic form and antigen uptake and presentation functions. The $\mathrm{CDC}$ are of two general types. Migratory $\mathrm{CDC}$, such as the Langerhans cells of the epidermis, begin as antigen col- 
lecting cells in peripheral tissues, then migrate through the lymphatics into lymph nodes where they present the antigens to $\mathrm{T}$ cells. Lymphoid tissue resident $\mathrm{cDC}$, which arrive in lymphoid tissues as blood-borne precursor cells, carry out both their antigen collecting and antigen presentation functions within the lymphoid organs. Finally, there are functionally distinct subsets of both migratory and tissue resident DCs (Shortman and Naik, 2007; Villadangos and Schnorrer, 2007). In the mouse, the latter can be segregated into CD8 ${ }^{+}$ and $\mathrm{CD}^{-}$subtypes. The $\mathrm{CD}^{+} \mathrm{CDC}$ are the major producers of IL-12p70 on activation (Reis e Sousa et al., 1997; Hochrein et al., 2001), so can initiate inflammatory Th1 type responses. They also have a special ability to take up dead cells and other material and cross-present these exogenous antigens on MHC class I (den Haan et al., 2000; Pooley et al., 2001; Schnorrer et al., 2006; Villadangos and Schnorrer, 2007). Thus, although they can activate CD4 $\mathrm{T}$ cells, they are especially adept at activating CD8 $T$ cells to produce cytotoxic $T$ cells. The CD8 ${ }^{-} \mathrm{CDC}$ are more adept at activating CD4 T cells (Dudziak et al., 2007; Villadangos and Schnorrer, 2007). These divisions, now well developed for the mouse DC system, are not yet fully established for the human DC system. In particular, CD8 $\alpha$ is not expressed on human DC so other markers may serve to distinguish important subsets. There is evidence that the BDCA $-3^{+} \mathrm{DC}$ subtype of human blood may represent a DC lineage equivalent to the mouse $C D 8^{+} \mathrm{CDC}$, since they have several other surface markers in common, including Necl2 (Galibert et al., 2005) and Clec9A (Caminschi et al., 2008; Huysamen et al., 2008; Sancho et al., 2008).

\section{Logistics of targeting antigens to DC}

Our increasing knowledge of the DC system should provide some guidance to the most effective targeting approach. Logically the DC surface molecule to be targeted should be as DC-specific as possible, to reduce the dose of antigen required. Binding to other cells could "mop up" the injected vaccine, as well as cause unwanted side effects. Unfortunately, there are few if any DCspecific surface molecules; even the mouse "DCmarker" molecule CD11c is expressed by other cells, including macrophages, NK cells and activated CD8 T cells. However, DC are strategically placed within the tissues so as to have selective access to introduced antigens, which compensates somewhat for reduced target specificity. Ideally the surface molecule targeted should be an endocytic receptor, and there is evidence that different receptors can shuttle antigens into different processing pathways (Burgdorf et al., 2007). The DC subtype presenting the antigen could determine the type of immune response obtained, so targeting specific DC subtypes should be an advantage; however we do not as yet understand all the rules governing DC subtype responses, especially in the human DC system. If the objective is to improve antibody responses, DC targeting should aim to maximise helper CD4 T cell responses which usually are the limiting factor; however it is unclear whether the eventual antibody producing B-cells should also be targeted, whether a little antigen will suffice for the $B$ cells, or whether the targeted DC can present antigen to the B cells as well as the helper $T$ cells (Wykes et al., 1998). Finally, since the eventual aim is to apply this targeting approach to humans, there is an advantage when working with mouse models to choose DC surface molecules common to the human and mouse DC systems.

Clearly there are many complex factors involved in DC targeting and currently it is difficult to predict how they will all play out in practice. At present there is a need to collect experimental data and learn some of the overriding rules, before applying this approach to clinical practice.

\section{The balance between immunity and tolerance}

Activation of the immune system can under some conditions lead to immune tolerance rather than active immunity. Finkelman (Finkelman et al., 1996) first noted that targeting antigen to the DC surface molecule now called DCIR2, using the $m A b$ 33D1, resulted in tolerance if no DC activating agents or adjuvants were also administered. This effect was then well documented by studies from the laboratories of Steinman and Nussenzweig, who targeted the multilectin receptor DEC-205 or CD205 (Hawiger et al., 2001; Bonifaz et al., 2002, 2004; Boscardin et al., 2006), that it is expressed by the $C D 8^{+} \mathrm{CDC}$ of mouse spleen and also by migratory $D C$ in lymph nodes. Administration of antigens linked to mAb against DEC-205 to steadystate DC produced MHC class I and MHC class II presentation and transient antigen- specific $T$ cell proliferation. However, antigen-specific CD4 and CD8 $T$ cells were subsequently deleted and regulatory CD4 T cells (Tregs) were generated (Hawiger et al., 2001; Bonifaz et al., 2002; Kretschmer et al., 2005; Yamazaki et al., 2008). The result was a failure to produce immune effector cells and the mice were unresponsive to further antigen cha- 
llenge, even with adjuvants. This approach has been shown to prevent the onset and even the progression of autoimmune diseases, by targeting autoantigens to DEC-205 in experimental mouse models of type 1 diabetes (Bruder et al., 2005; Mukhopadhaya et al., 2008).

If the objective in targeting antigens to $D C$ is to produce a vaccine giving both enhanced immune responses and effective immunological memory, then the factors which induce tolerance must be avoided or negated. The extensive studies on DEC-205 targeting indicated that co-administration of factors which activate DC (such as anti-CD40 or toll-like receptor ligands) permits effective cellular and immune responses to the antigens coupled to anti-DEC-205 mAb (Bonifaz et al., 2004; Boscardin et al., 2006; Soares et al., 2007; Trumpfheller et al., 2008). These studies have supported the generalisation that targeting antigens to steady-state, non-activated DC leads to tolerance whereas targeting to DC simultaneously activated with adjuvants produces immunity. As discussed below, this concept fits most of the findings on targeting to produce CTL responses, but it is not always in accord with the studies on antibody responses.

\section{Enhancing CTL responses by targeting antigens to DC}

Effective generation of cellular immunity, particularly of CTL, is required for resistance to some viral infections and is a major requirement in tumor immunotherapy. CTL responses have been generated by targeting antigens to a range of $D C$ surface molecules, including DEC-205 (Hawiger et al., 2001; Bonifaz et al., 2002, 2004), DCIR2 (Dudziak et al., 2007; Soares et al., 2007), mannose receptor (He et al., 2007), Dectin-1 (Carter et al., 2006), Clec9A (Sancho et al., 2008), CD11c and MHC class II (Castro et al., 2008). In all these cases some form of adjuvant or DC activating agent was employed to obtain effective cellular immunity, suggesting this is a general requirement for CTL generation. One apparent exception to this was targeting antigens to the scavenger receptor CD36, where without adjuvants a CD8 T cell response was generated and this was associated with a prevention of tumour growth (Tagliani et al., 2008). It needs to be verified that inadvertent activation of DC by microbial products had not occurred, before targeting CD36 can be considered as an exceptional case.

In theory targeting antigens to the specialised "cross-presenting" CD8 ${ }^{+}$cDC subtype in mice, or its proposed equivalent in the human DC system, should produce optimal CD8 T cell responses and CTL generation. Targeting DEC-205 or Clec9A should accomplish this directly, and they have proved effective for this purpose (Hawiger et al., 2001; Bonifaz et al., 2002, 2004; Sancho et al., 2008). However, $\mathrm{CD}^{+} \mathrm{CDC}$ have a capacity to collect and process antigen from dead cells (lyoda et al., 2002; Schulz and Reis e Sousa, 2002; Schnorrer et al., 2006), so even antigens targeted to other DC may eventually be reprocessed by $\mathrm{CD}^{+} \mathrm{CDC}$. An additional advantage of targeting $\mathrm{CD}^{+} \mathrm{CDC}$ is that these are the major producers of IL-12p70 (Reis e Sousa et al., 1997; Hochrein et al., 2001), a well established factor for CTL development. However, IL-12p70 is only produced when $\mathrm{CD}^{+} \mathrm{CDC}$ are activated, one reason why adjuvants or DC activation agents are required for $C T L$ generation.

\section{Enhancing antibody responses by targeting antigens to DC}

We are particularly interested in improving antibody responses, since there are many situations where possible vaccine antigens give only limited protection against infections because of low antibody production. Our assumption is that helper $\mathrm{T}$ cells were the limiting factor, so targeting antigens to $D C$ should enhance the response of CD4 T cells and indirectly improve antibody production. Targeting antigens to many different DC surface molecules has enhanced CD4 T cell responses (Hawiger et al., 2001; Bonifaz et al., 2002; Bonifaz et al., 2004; Carter et al., 2006; Dudziak et al., 2007; He et al., 2007; Soares et al., 2007; Caminschi et al., 2008; Castro et al., 2008). We have found enhanced antibody responses by targeting antigens to the DC surface molecules FIRE, CIRE, Clec9A and Clec12A, and shown this response is dependent on $\mathrm{T}$ cells and on MHC class II antigen presentation by DC ((Corbett et al., 2005; Caminschi et al., 2008); Caminschi, unpublished). Targeting CD8 ${ }^{\circ} \mathrm{CDC}$ would seem the most effective strategy for obtaining optimal CD4 $\mathrm{T}$ cell responses, and enhanced antibody responses have been obtained by this approach (Corbett et al., 2005). However, CD ${ }^{+} \mathrm{CDC}$ are capable of effective CD4 $\mathrm{T}$ cell activation despite deflection of much antigen into the MHC class I presentation pathway (Pooley et al., 2001; Schnorrer et al., 2006), and excellent antibody responses have been obtained by targeting antigen to DEC-205 (Boscardin et al., 2006; Soares et al., 2007) or to Clec9A (Caminschi et al., 2008) which are selectively expressed by CD8 ${ }^{+}$ cDC. 
An important issue is whether DC activating agents or adjuvants are required to obtain enhanced antibody responses, as they are for CTL responses. Avoiding the side effects of adjuvants would be a step forward in vaccine design. In the standard setting studies targeting antigens to DEC-205 (Boscardin et al., 2006), simultaneous DC activation was required to obtain antibody production, just as for CTL production; our own earlier studies targeting DEC-205 gave the same result (Corbett et al., 2005). In line with this finding, adjuvants were required for good antibody production when targeting antigens to Dectin-1 (Carter et al., 2006) and DCIR2 (Finkelman et al., 1996). However, in a series of other studies targeting antigens to other molecules on DC, strong antibody responses were obtained without using adjuvants; the molecules targeted included $\mathrm{MHC}$ class II (Carayanniotis and Barber, 1987); MHC class I, FcR $\gamma$ Il (Snider et al., 1990), CD45, CD45RA, DCIR-2, CD4 (Skea and Barber, 1993), the mannose receptor (He et al., 2007) and from our laboratory CIRE (mDC SIGN), FIRE (Corbett et al., 2005) and Clec9A (Caminschi et al., 2008). In the latter case the same result was obtained using

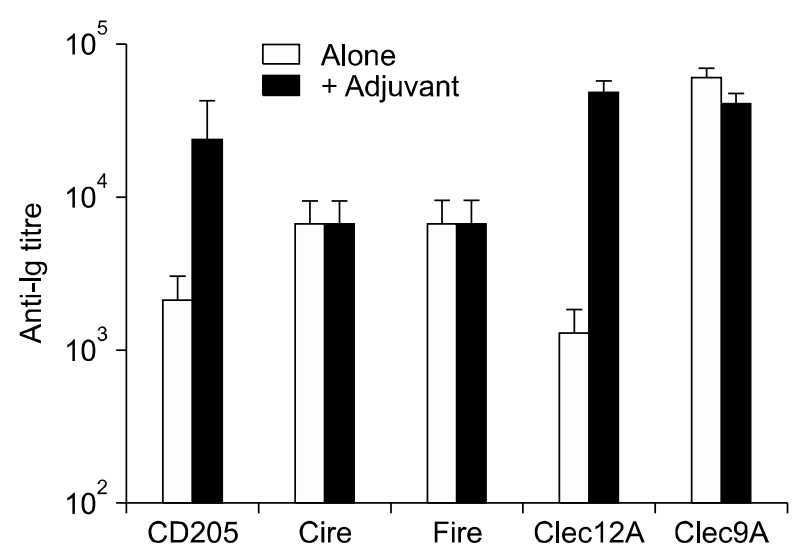

Figure 1. Are adjuvants needed to obtain antibody responses on targeting antigens to $D C$ ? The results presented summarise our experience in targeting antigen to $D C$ by intraveneous injection into mice of rat $\mathrm{mAb}$ against mouse DC surface molecules. The readout is the mouse antibody response to rat immunoglobulin. In all cases a non-targeting isotype-matched rat lg gave negligible responses in the absence of adjuvants. Similar results have been obtained for the anti-ovalbumin response when ovalbumin was linked to the targeting mAb. The adjuvants or DC activating agents used have included lipopolysaccharide, poly I:C, $\mathrm{CpG}$ and alum; all gave similar effects although the efficiency varied. Although these experiments were not conducted side by side, the results have been consistent once a common sensitive ELISA assay for antibody production was employed. Note that DEC-205 and Clec9A are selectively expressed by $\mathrm{CD} 8^{+} \mathrm{DC}$, Fire and Cire are selectively expressed by $C D 8^{-} \mathrm{DC}$, while Clec12A is on all DC but highest on $\mathrm{CD8^{+ }} D \mathrm{D}$. Thus the results depend more on the DC surface molecule targeted than the DC subtype.
MyD88 $^{-/-} \mathrm{TRIF}^{-/-}$mice which are unable to respond to toll-like receptor ligands, so DC activation by contaminating microbial products was not the reason. What are the rules which determine whether an adjuvant is required for antibody production?

Our experience on this issue is summarised in Figure 1. With a more sensitive antibody assay we now always see some antibody production on targeting antigens to all DC molecules tested. However, adjuvants markedly enhance antibody production on targeting DEC-205 or targeting Clec12A. In contrast, good antibody production is obtained without adjuvants on targeting antigens to CIRE, FIRE and Clec9A. Since CIRE and FIRE are on CD8 ${ }^{-D C}$ and Clec9A, like DEC-205, is on $C D 8^{+} D C$, it seems the subtype of DC targeted is not the determining factor. Rather, the nature of the DC surface molecule targeted determines whether the response requires $D C$ activation. It is possible that DC surface molecules like FIRE, CIRE and Clec9A transmit a subtle signal which, without inducing all the features of DC activation, promotes production of the cytokines required to produce the appropriate helper T cells. Alternatively, these molecules may shuttle antigens into a different processing pathway, perhaps allowing effective antigen presentation to both helper T cells and B cells. The basic mechanism needs to be understood before this promising approach to adjuvant free vaccines can be applied to human populations.

\section{Clec9A as a vaccine}

The promise of DC targeting for enhancing the effectiveness of vaccines is well exemplified in recent studies targeting Clec9A. This DC surface molecule, recently described by three laboratories (Caminschi et al., 2008; Huysamen et al., 2008; Sancho et al., 2008), is a C-type lectin-like molecule with a higher specificity for DCs than most DC markers. It is expressed on the $C D 8^{+} \mathrm{CDC}$ subtype in mice and the equivalent Clec9A molecule is expressed on the BDCA-3 DC subtype in man, the proposed equivalent of the mouse CD8 ${ }^{+}$ $\mathrm{cDC}$ lineage. Several mAb against both the mouse and human Clec9A are available for target studies. Sancho et al. (2008) have shown that targeting tumor antigens to Clec9A, along with DC activation agents, promotes $T$ cell responses, CTL production and effective rejection of tumours. Our laboratory has shown that, even without adjuvants, targeting Clec9A promotes the best antibody responses we have obtained (Figure 1) and requires only tiny amounts of the targeting $\mathrm{mAb}$ vaccine (Caminschi 
et al., 2008). Some clues to why Clec9A is so effective as a target have come from the Reis e Sousa group (personal communication) and from our laboratory (Lahoud et al., unpublished). Clec9A serves as a recognition molecule for dead cells. The $C D 8^{+} \mathrm{CDC}$, which express Clec9A are especially adept at taking up dead cells and reprocessing dead cell antigens (lyoda et al., 2002; Schulz and Reis e Sousa, 2002; Schnorrer et al., 2006). Thus targeting antigens to Clec9A via antiClec9A mAb appears to shunt antigens directly into a normal biological process for efficient processing of exogenous antigens. The existence of the equivalent CLEC9A molecule on the surface of a specific subtype of human DC (Caminschi et al., 2008; Huysamen et al., 2008; Sancho et al., 2008) suggests that, once the mouse model studies are complete, translation to human trials could be rapid.

\section{References}

Bonifaz L, Bonnyay D, Mahnke K, Rivera M, Nussenzweig MC, Steinman RM. Efficient targeting of protein antigen to the dendritic cell receptor DEC-205 in the steady state leads to antigen presentation on major histocompatibility complex class I products and peripheral CD8+ T cell tolerance. J Exp Med 2002;196:1627-38

Bonifaz LC, Bonnyay DP, Charalambous A, Darguste DI, Fujii S, Soares H, Brimnes MK, Moltedo B, Moran TM, Steinman RM. In vivo targeting of antigens to maturing dendritic cells via the DEC-205 receptor improves T cell vaccination. J Exp Med 2004;199:815-24

Boscardin SB, Hafalla JC, Masilamani RF, Kamphorst AO, Zebroski HA, Rai U, Morrot A, Zavala F, Steinman RM, Nussenzweig RS, Nussenzweig MC. Antigen targeting to dendritic cells elicits long-lived T cell help for antibody responses. J Exp Med 2006;203:599-606

Bruder D, Westendorf AM, Hansen W, Prettin S, Gruber AD, Qian Y, von Boehmer H, Mahnke K, Buer J. On the edge of autoimmunity: T-cell stimulation by steady-state dendritic cells prevents autoimmune diabetes. Diabetes 2005;54: 3395-401

Burgdorf S, Kautz A, Bohnert V, Knolle PA, Kurts C. Distinct pathways of antigen uptake and intracellular routing in CD4 and CD8 T cell activation. Science 2007;316:612-6

Caminschi I, Proietto AI, Ahmet F, Kitsoulis S, Shin Teh J, Lo JC, Rizzitelli A, Wu L, Vremec D, van Dommelen SL, et al. The dendritic cell subtype-restricted C-type lectin Clec9A is a target for vaccine enhancement. Blood 2008;112:3264-73

Carayanniotis $\mathrm{G}$, Barber $\mathrm{BH}$. Adjuvant-free IgG responses induced with antigen coupled to antibodies against class II MHC. Nature 1987;327:59-61

Carter RW, Thompson C, Reid DM, Wong SY, Tough DF. Preferential induction of CD4+ T cell responses through in vivo targeting of antigen to dendritic cell-associated C-type lectin-1. J Immunol 2006;177:2276-84

Castro FV, Tutt AL, White AL, Teeling JL, James S, French RR, Glennie MJ. CD11c provides an effective immunotarget for the generation of both CD4 and CD8 T cell responses. Eur $\mathrm{J}$ Immunol 2008;38:2263-73

Corbett AJ, Caminschi I, McKenzie BS, Brady JL, Wright MD, Mottram PL, Hogarth PM, Hodder AN, Zhan Y, Tarlinton DM, et al. Antigen delivery via two molecules on the CD8- dendritic cell subset induces humoral immunity in the absence of conventional "danger". Eur J Immunol 2005;35:2815-25

den Haan JM, Lehar SM, Bevan MJ. CD8(+) but not CD8(-) dendritic cells cross-prime cytotoxic T cells In vivo. J Exp Med 2000;192:1685-96

Dudziak D, Kamphorst AO, Heidkamp GF, Buchholz VR, Trumpfheller C, Yamazaki S, Cheong C, Liu K, Lee HW, Park CG, et al. Differential antigen processing by dendritic cell subsets in vivo. Science 2007;315:107-11

Finkelman FD, Lees A, Birnbaum R, Gause WC, Morris SC. Dendritic cells can present antigen in vivo in a tolerogenic or immunogenic fashion. J Immunol 1996;157:1406-14

Galibert L, Diemer GS, Liu Z, Johnson RS, Smith JL, Walzer T, Comeau MR, Rauch CT, Wolfson MF, Sorensen RA, et al. Nectin-like protein 2 defines a subset of T-cell zone dendritic cells and is a ligand for class-I-restricted T-cell-associated molecule. J Biol Chem 2005;280:21955-64

Hawiger D, Inaba K, Dorsett Y, Guo M, Mahnke K, Rivera M, Ravetch JV, Steinman RM, Nussenzweig MC. Dendritic cells induce peripheral $T$ cell unresponsiveness under steady state conditions in vivo. J Exp Med 2001;194:769-79

He LZ, Crocker A, Lee J, Mendoza-Ramirez J, Wang XT, Vitale LA, O'Neill T, Petromilli C, Zhang HF, Lopez J, et al. Antigenic targeting of the human mannose receptor induces tumor immunity. J Immunol 2007;178:6259-67

Hochrein H, Shortman K, Vremec D, Scott B, Hertzog P, O'Keeffe M. Differential production of IL-12, IFN-alpha, and IFN-gamma by mouse dendritic cell subsets. J Immunol 2001;166:5448-55

Huysamen C, Willment JA, Dennehy KM, Brown GD. CLEC9A is a novel activation C-type lectin-like receptor expressed on BDCA3+ dendritic cells and a subset of monocytes. J Biol Chem 2008.

lyoda T, Shimoyama S, Liu K, Omatsu Y, Akiyama Y, Maeda Y, Takahara K, Steinman RM, Inaba K. The CD8+ dendritic cell subset selectively endocytoses dying cells in culture and in vivo. J Exp Med 2002;195:1289-302

Kretschmer K, Apostolou I, Hawiger D, Khazaie K, Nussenzweig MC, von Boehmer $\mathrm{H}$. Inducing and expanding regulatory $T$ cell populations by foreign antigen. Nat Immunol 2005;6:1219-27

Mukhopadhaya A, Hanafusa T, Jarchum I, Chen YG, Iwai Y, Serreze DV, Steinman RM, Tarbell KV, Dilorenzo TP. Selective delivery of $\beta$ cell antigen to dendritic cells in vivo leads to deletion and tolerance of autoreactive CD8+ T cells in NOD mice. Proc Natl Acad Sci USA 2008.

Pooley J, Heath WR, Shortman K. Intravenous soluble antigen is presented to CD4 T cells by CD8- Dendritic cells 
but cross-presented to CD8+ T cells by CD8+ Dendritic cells. $\mathrm{J}$ Immunol 2001;166:5327-30

Reis e Sousa C, Hieny S, Scharton-Kersten T, Jankovic D, Charest $\mathrm{H}$, Germain RN, Sher A. In vivo microbial stimulation induces rapid CD40 ligand-independent production of interleukin 12 by dendritic cells and their redistribution to $T$ cell areas. J Exp Med 1997;186:1819-29

Sancho D, Mourao-Sa D, Joffre OP, Schulz O, Rogers NC, Pennington DJ, Carlyle JR, Reis e Sousa C. Tumor therapy in mice via antigen targeting to a novel, DC-restricted C-type lectin. J Clin Invest 2008;118:2098-110

Schnorrer P, Behrens GM, Wilson NS, Pooley JL, Smith CM, El-Sukkari D, Davey G, Kupresanin F, Li M, Maraskovsky E, et al. The dominant role of $\mathrm{CD} 8+$ dendritic cells in cross-presentation is not dictated by antigen capture. Proc Natl Acad Sci USA 2006;103:10729-34

Schulz O, Reis e Sousa C. Cross-presentation of cellassociated antigens by CD8alpha+ dendritic cells is attributable to their ability to internalize dead cells. Immunology 2002;107:183-9

Shortman K, Naik SH. Steady-state and inflammatory dendritic-cell development. Nat Rev Immunol 2007;7:19-30

Skea DL, Barber BH. Studies of the adjuvant-independent antibody response to immunotargeting. Target structure dependence, isotype distribution, and induction of long term memory. J Immunol 1993;151:3557-68

Snider DP, Kaubisch A, Segal DM. Enhanced antigen immunogenicity induced by bispecific antibodies. J Exp Med 1990;171:1957-63

Soares H, Waechter H, Glaichenhaus N, Mougneau E, Yagita H, Mizenina O, Dudziak D, Nussenzweig MC, Stein- man RM. A subset of dendritic cells induces CD4+ T cells to produce IFN-gamma by an IL-12-independent but CD70dependent mechanism in vivo. J Exp Med 2007;204: 1095-106

Steinman RM. The dendritic cell system and its role in immunogenicity. Annu Rev Immunol 1991;9:271-96

Tacken PJ, de Vries IJ, Torensma R, Figdor CG. Dendritic-cell immunotherapy: from ex vivo loading to in vivo targeting. Nat Rev Immunol 2007;7:790-802

Tagliani E, Guermonprez P, Sepulveda J, Lopez-Bravo M, Ardavin C, Amigorena S, Benvenuti F, Burrone OR. Selection of an antibody library identifies a pathway to induce immunity by targeting CD36 on steady-state CD8alpha+ dendritic cells. J Immunol 2008;180:3201-9

Trumpfheller C, Caskey M, Nchinda G, Longhi MP, Mizenina $\mathrm{O}$, Huang Y, Schlesinger SJ, Colonna M, Steinman RM. The microbial mimic poly IC induces durable and protective CD4+ $T$ cell immunity together with a dendritic cell targeted vaccine. Proc Natl Acad Sci USA 2008;105:2574-9

Villadangos JA, Schnorrer P. Intrinsic and cooperative antigen-presenting functions of dendritic-cell subsets in vivo. Nat Rev Immunol 2007;7:543-55

Wykes M, Pombo A, Jenkins C, MacPherson GG. Dendritic cells interact directly with naive $B$ lymphocytes to transfer antigen and initiate class switching in a primary T-dependent response. J Immunol 1998;161:1313-9

Yamazaki S, Dudziak D, Heidkamp GF, Fiorese C, Bonito AJ, Inaba K, Nussenzweig MC, Steinman RM. CD8+ CD205+ splenic dendritic cells are specialized to induce Foxp3+ regulatory T cells. J Immunol 2008;181:6923-33 